Original Research Article

\title{
Clinical profiling of patients with Acid Peptic Disorders (APD) in India: a cross-sectional survey of clinicians
}

\author{
Ramesh Roop Rai $^{1}$, A. Gangadhar ${ }^{2}$, Mayur M. Mayabhate ${ }^{3 *}$
}

\author{
${ }^{1}$ Rai Specialty Care Centre, \\ Jaipur, H-6, Janpath, Shyam \\ Nagar, Jaipur, Rajasthan, India \\ ${ }^{2}$ Abbott Healthcare Pvt Ltd, \\ Mumbai, Maharashtra, India \\ ${ }^{3}$ Abbott India Limited, Mumbai, \\ Maharashtra, India
}

Received: 14 November 2016 Accepted: 09 December 2016

*Correspondence to: Dr. Mayur M. Mayabhate, Email: mayur.mayabhate@abbott.com

Copyright: (C) the author(s), publisher and licensee Medip Academy. This is an openaccess article distributed under the terms of the Creative Commons Attribution NonCommercial License, which permits unrestricted noncommercial use, distribution, and reproduction in any medium, provided the original work is properly cited.

\begin{abstract}
Background: Acid peptic disorders (APD) which include GERD and peptic ulcer disease (PUD) are common conditions reported in daily clinical practice. This survey aimed to understand the epidemiology, clinical presentation and associated overlapping comorbidities in Indian patients with APD.

Methods: This was an interview based cross-sectional survey of 1000 clinicians (specialist gastroenterologists and non-specialists) across India who treated patients of APD in their practice. Information related to patient demographics, diagnosis and clinical presentation (common symptoms and their duration, alarming, lower GI and extra-esophageal symptoms) were assessed. Indications for upper GI endoscopy, esophageal $\mathrm{pH}$ monitoring and esophageal manometry were also collected. Descriptive analyses were done.

Results: About $39.2 \%$ and $37.1 \%$ patients had reported GERD and PUD respectively (duodenal ulcer: $10.5 \%$, gastric ulcer: $9.9 \%$ and peptic ulcer-nonspecified: $16.7 \%) ; 25.2 \%$ patients were reported to have non-ulcer dyspepsia. Heartburn was the most common symptom in GERD (60.5\%); epigastric pain was common in PUD (72.3\%); 49\% GERD patients presented with alarming symptoms, mainly dysphagia (67\%), whereas for PUD, GI bleeding was the most common alarming symptom (47.6\%). Profiles of patients visiting clinicians were similar with respect to the presenting symptoms and associated conditions. Functional dyspepsia (25.9\%), constipation $(23.4 \%)$ and irritable bowel syndrome $(23.4 \%)$ were the most common overlapping conditions associated with both GERD and PUD.

Conclusions: APD (GERD and PUD) was more common in 18-59 yrs. age group with heart burn as the common symptom for GERD and epigastric pain for PUD. Diagnosis was mostly based on clinical symptoms; nearly 50\% patients of APD presenting with alarming symptoms needed prompt endoscopy. Thus, there is need to focus on these overlapping disorders while managing patients of APD.
\end{abstract}

Keywords: Alarming symptoms, GERD, Heartburn, Overlapping conditions

\section{INTRODUCTION}

Acid peptic disorders (APD) are the result of distinctive, but overlapping pathogenic mechanisms leading to either excessive acid secretion or diminished mucosal defense. It usually includes two conditions: gastroesophageal reflux disease (GERD) and peptic ulcer disease (PUD). ${ }^{1}$ However, other conditions like functional dyspepsia and irritable bowel syndrome (IBS) commonly overlap in the patients with APD making the diagnosis difficult. Hence it would be useful to understand the approach of specialist (SS) and non-specialist (NSS) clinicians during the management of APD. To exclude the other overlapping conditions, investigations like upper GI scopy, esophageal manometry are commonly used by specialist gastroenterologists.

An epidemiology study in 30,216 patients $(41.7 \pm 12.7$ years; $66 \%$ males) reported a prevalence of $7.8 \%$ for PUD in India. ${ }^{2}$ The report of the Indian Society of Gastroenterology Task Force highlights that $7.6 \%$ of Indian subjects have significant GERD symptoms. Consumption of non-vegetarian foods was an independent predictor of GERD. ${ }^{3}$ The prevalence of GERD in patients at respiratory clinic at a tertiary care hospital from south-west India was reported to be around $28 \%{ }^{4}$ The common presenting features of GERD in these patients were heartburn (18.9\%) retrosternal pain $(6.8 \%)$, 
dyspepsia (5.2\%), regurgitation $(1.7 \%)$ and extraesophageal manifestation as nocturnal cough (5.2\%). Thus, the prevalence of GERD in India is likely to be between $8 \%$ and $19 \%$ in general population, which is comparable to GERD prevalence rates published in western countries where the prevalence of $15-20 \%$ is being reported. ${ }^{5}$ PUD tends to have a chronic remitting course with imperfect correlation between symptoms and the presence of an ulcer, and the annual incidence rates of PUD are $0.10-0.19 \%$ for physician-diagnosed PUD and $0.03-0.17 \%$ when based on hospitalization data, whereas the 1-year prevalence is reported to be up to $1.5 \% .^{6} \mathrm{~A}$ study by Aro P. et al, reported a $4.1 \%$ prevalence of PUD ( $2 \%$ gastric ulcers and $2.1 \%$ duodenal ulcers). ${ }^{7}$ In another retrospective study involving 10,059 patients undergoing endoscopy from southern India conducted between January 2009 to December 2011, the reported prevalence of GERD was $30 \%$, with a male: female ratio of $1.8: 1 .^{8}$

Although the pathophysiology of GERD and PUD has been studied extensively, literature evidence reporting clinical presentation of patients with APD (GERD and PUD) in India is lacking. Moreover, an approach to APD patient might differ between specialist and non-specialist clinician. This clinician based survey was conducted across India to understand the epidemiology, clinical presentation and common overlapping conditions in patients of APD. The study also tried to understand whether any differences exist in terms of the patients visiting specialists (gastroenterologists) and nonspecialists (general practitioners and consultants) and their clinical presentations.

\section{METHODS}

\section{Study design and setting}

The interview based cross-sectional survey of clinicians was conducted across all the geographic regions (urban and semi-urban areas) of India (Figure 1). Clinicians with $>3$ years of clinical practice, treating patients of APD on a regular basis and willing to participate were included in this study. The responders included general practitioners (114), consulting physicians (236), general surgeons (92), consulting gastro-physicians (278), specialist gastroenterologists (223) and gastrosurgeons (57). The clinicians involved in the survey were from private clinics (438), government hospitals (200), corporate hospitals (302) and other settings (60). As this survey does not involve the direct participation of any patient, this study was not submitted to ethics committee for approval.

The study was conducted in accordance with the protocol, ethical principles having their origin in the Declaration of Helsinki, International Conference on Harmonization Good Clinical Practice (ICH-GCP) guidelines, all applicable government regulations and Institutional research policies and procedures. All the information collected during study was kept confidential.

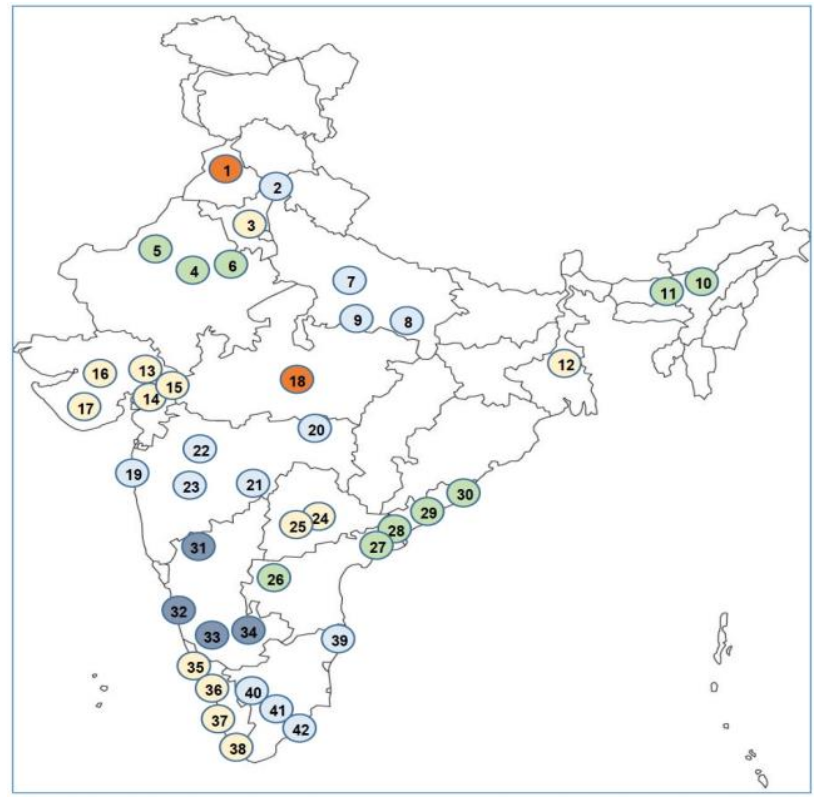

Figure 1: Distribution of participating clinician's across India $(\mathbf{n}=\mathbf{1 0 0 0})$.

\begin{tabular}{|llllll|}
\hline Sr. & City & $\#$ & Sr. & City & $\#$ \\
\hline 1 & Jalandhar & 1 & 22 & Nashik & 9 \\
\hline 2 & Delhi & 85 & 23 & Pune & 44 \\
\hline 3 & Gurgaon & 8 & 24 & Hyderabad & 79 \\
\hline 4 & Ajmer & 1 & 25 & Secunderabad & 2 \\
\hline 5 & Bikaner & 7 & 26 & Anantpur & 1 \\
\hline 6 & Jaipur & 41 & 27 & Guntur & 5 \\
\hline 7 & Lucknow & 54 & 28 & Vijayawada & 6 \\
\hline 8 & Allahabad & 1 & 29 & Rajahmundry & 1 \\
\hline 9 & Kanpur & 1 & 30 & Visakhapatnam & 24 \\
\hline 10 & Assam & 1 & 31 & Belgaum & 3 \\
\hline 11 & Guwahati & 21 & 32 & Mangalore & 4 \\
\hline 12 & Kolkata & 20 & 33 & Mysore & 206 \\
\hline 13 & Ahmedabad & 33 & 34 & Bangalore & 65 \\
\hline 14 & Baroda & 13 & 35 & Calicut & 4 \\
\hline 15 & Godhra & 1 & 36 & Kerala & 9 \\
\hline 16 & Gujarat & 6 & 37 & Kochi & 3 \\
\hline 17 & Rajkot & 2 & 38 & Trivandrum & 7 \\
\hline 18 & Bhopal & 12 & 39 & Chennai & 49 \\
\hline 19 & Mumbai & 81 & 40 & Coimbatore & 27 \\
\hline 20 & Nagpur & 23 & 41 & Madurai & 44 \\
\hline 21 & Nanded & 1 & 42 & Shivaganga & 1 \\
\hline & & & & & \\
\hline
\end{tabular}

\section{Data collection}

Clinicians were personally interviewed by trained personnel and their responses were captured in the study specific structured questionnaire. The clinicians were asked to recall the information of patients with APD whom they had treated. Information related to patient demographics, diagnosis (GERD, gastric ulcer, duodenal ulcer and non-ulcer dyspepsia) and clinical presentation (common symptoms, duration of clinical symptoms, alarming symptoms, lower gastrointestinal symptoms and 
extra-esophageal symptoms) was collected. The symptoms were ranked by the responders from 1 to 5 where rank 1 was the 'most common symptom' and rank 5 was the 'least common symptom'. The typical symptoms, alarming symptoms and common overlapping conditions associated with GERD and PUD were also collected. The information regarding the diagnostic criteria used by clinicians for diagnosis of GERD and PUD, indications for upper GI endoscopy, indications for esophageal $\mathrm{pH}$ monitoring and indications for esophageal manometry were also collected. The proportion of erosive and non-erosive GERD in different age groups and percentage of patients encountering alarming symptoms in GERD and PUD were also collected.

\section{Statistical methods}

\section{Sample size}

Based on the reported data that $46 \%$ physicians prescribed acid suppressant drugs for GERD, a sample size of 500 yields a half-width of at most 0.05 with a conditional probability of 1 for a two-sided $95 \%$ exact Clopper-Pearson confidence interval for a binomial proportion whose true value is $0.46 .{ }^{9}$ Hence, it was planned to include a total of 1000 clinicians (500 specialists and 500 non-specialists) who had treated patients with GERD and/or PUD for the survey. Although no formal sampling technique was used, an attempt was made to enroll equal proportion of clinicians from different regions of India so that the survey results are comprehensive and represent the entire country.

\section{Statistical analyses}

Standard descriptive statistics were applied to the data collected. The summary of categorical data was presented in terms of counts (n) and percentages (\%). All continuous variables were described in terms of counts, mean, standard deviation (SD), median, minimum and maximum. The study being a survey, there were no hypothesis and comparisons done between groups and sub-groups.

\section{RESULTS}

\section{Responder profile}

The profile of responder clinicians is presented in Table 1

Table 1: Responder (clinicians) profile.

\begin{tabular}{|c|c|c|c|c|c|c|c|c|c|c|}
\hline & & \multicolumn{4}{|c|}{ Non-specialists (NS) } & \multicolumn{4}{|c|}{ Specialists (SS) } & \multirow[t]{2}{*}{ Overal } \\
\hline & & $\begin{array}{l}\text { General } \\
\text { Practitioner }\end{array}$ & $\begin{array}{l}\text { General } \\
\text { Physician }\end{array}$ & $\begin{array}{l}\text { General } \\
\text { Surgeon }\end{array}$ & $\begin{array}{l}\text { Total } \\
\text { (NS) }\end{array}$ & $\begin{array}{l}\text { Gastro- } \\
\text { Physician }\end{array}$ & $\begin{array}{l}\text { Gastro- } \\
\text { Enterologist }\end{array}$ & $\begin{array}{l}\text { Gastro- } \\
\text { Surgeon }\end{array}$ & $\begin{array}{l}\text { Total } \\
\text { (SS) }\end{array}$ & \\
\hline & $\mathrm{N}$ & 114 & 236 & 92 & 442 & 278 & 223 & 57 & 558 & 1000 \\
\hline \multicolumn{11}{|c|}{ Years of clinical experience } \\
\hline$<5$ years & $\mathrm{n}(\%)$ & $30(26.3)$ & $54(22.9)$ & $15(26.3)$ & $\begin{array}{l}99 \\
(22.4)\end{array}$ & $112(40.3)$ & $65(29.1)$ & $10(10.9)$ & $\begin{array}{l}187 \\
(33.5)\end{array}$ & $\begin{array}{l}286 \\
(28.6)\end{array}$ \\
\hline $\begin{array}{l}5-10 \\
\text { years }\end{array}$ & $\mathrm{n}(\%)$ & $45(39.5)$ & $97(41.1)$ & $31(54.4)$ & $\begin{array}{l}173 \\
(39.1)\end{array}$ & $92(33.1)$ & $82(36.8)$ & $14(15.2)$ & $\begin{array}{l}188 \\
(33.7)\end{array}$ & $\begin{array}{l}361 \\
(36.1)\end{array}$ \\
\hline $\begin{array}{l}11-20 \\
\text { years }\end{array}$ & $\mathrm{n}(\%)$ & $21(18.4)$ & $52(22.0)$ & $30(52.6)$ & $\begin{array}{l}103 \\
(23.3)\end{array}$ & $57(20.5)$ & $45(20.2)$ & $23(25.0)$ & $\begin{array}{l}125 \\
(22.4)\end{array}$ & $\begin{array}{l}228 \\
(22.8)\end{array}$ \\
\hline $\begin{array}{l}>20 \\
\text { years }\end{array}$ & $\mathrm{n}(\%)$ & $18(15.8)$ & $30(12.7)$ & $15(26.3)$ & $\begin{array}{l}63 \\
(14.3)\end{array}$ & $17(6.1)$ & $26(11.7)$ & $9(9.8)$ & $\begin{array}{l}52 \\
(9.3)\end{array}$ & $\begin{array}{l}115 \\
(11.5)\end{array}$ \\
\hline Missing & $\mathrm{n}(\%)$ & 0 & $3(1.3)$ & $1(1.8)$ & $\begin{array}{l}4 \\
(0.9)\end{array}$ & 0 & $5(2.2)$ & $1(1.1)$ & $\begin{array}{l}6 \\
(1.1)\end{array}$ & $10(1.0)$ \\
\hline \multicolumn{11}{|c|}{ Affiliation to the institutions } \\
\hline $\begin{array}{l}\text { Private } \\
\text { Clinic }\end{array}$ & $\mathrm{n}(\%)$ & $61(53.5)$ & $121(51.3)$ & $34(59.6)$ & $\begin{array}{l}216 \\
(48.9) \\
\end{array}$ & $142(51.1$ & $69(30.9)$ & $11(12.0)$ & $\begin{array}{l}222 \\
(39.8)\end{array}$ & $\begin{array}{l}438 \\
(43.8) \\
\end{array}$ \\
\hline $\begin{array}{l}\text { Govt. } \\
\text { hospital }\end{array}$ & $\mathrm{n}(\%)$ & $20(17.5)$ & $38(16.1)$ & 22 (38.6) & $\begin{array}{l}80 \\
(18.1)\end{array}$ & $53(19.1)$ & $54(24.2)$ & $13(14.1)$ & $\begin{array}{l}120 \\
(21.5)\end{array}$ & $\begin{array}{l}200 \\
(20.0)\end{array}$ \\
\hline $\begin{array}{l}\text { Corporate } \\
\text { hospital }\end{array}$ & $\mathrm{n}(\%)$ & $28(24.6)$ & $64(27.1)$ & $29(50.9)$ & $\begin{array}{l}121 \\
(27.4)\end{array}$ & $70(25.2)$ & $85(38.1)$ & $26(28.3)$ & $\begin{array}{l}181 \\
(32.4)\end{array}$ & $\begin{array}{l}302 \\
(30.2)\end{array}$ \\
\hline Others & $\mathrm{n}(\%)$ & $5(4.4)$ & $13(5.5)$ & $6(10.5)$ & $\begin{array}{l}24 \\
(5.4)\end{array}$ & $12(4.3)$ & $13(5.8)$ & $7(7.6)$ & $\begin{array}{l}32 \\
(5.7)\end{array}$ & $56(5.6)$ \\
\hline Missing & $\mathrm{n}(\%)$ & 0 & 0 & $1(1.8)$ & $\begin{array}{l}1 \\
(0.2)\end{array}$ & $1(0.4)$ & $2(0.9)$ & 0 & $\begin{array}{l}3 \\
(0.5)\end{array}$ & $4(0.4)$ \\
\hline \multicolumn{11}{|c|}{ Average number of patients the respondent treat in a week in routine practice (patients/week) } \\
\hline & $\mathrm{N}$ & 111 & 234 & 91 & 436 & 278 & 222 & 57 & 557 & 993 \\
\hline & Mean (SD) & 158.4 & 173.1 & 136.9 & 161.8 & 140.1 & 165.3 & 149.3 & 151.1 & 155.8 \\
\hline & SD & 105.9 & 129.1 & 97.7 & 116.6 & 97.3 & 148.6 & 106.7 & 118.7 & 120.1 \\
\hline & Median & 140 & 150 & 125 & 150 & 120 & 120 & 140 & 120 & 130 \\
\hline & $\begin{array}{l}\text { Min, } \\
\text { Max }\end{array}$ & 15,500 & 10,1000 & 10,600 & $\begin{array}{l}10 \\
1000\end{array}$ & 10,700 & 10,1000 & 25,600 & $\begin{array}{l}10 \\
1000\end{array}$ & 10,1000 \\
\hline
\end{tabular}


There were mainly specialist gastroenterologists and consulting physicians. There were few surgeons (NSS group: $20.81 \%$; SS group: $10.21 \%$ ) compared to physicians. Many clinicians were having experience of over 5 years, and about $14.3 \%$ doctors from NSS and 9.3\% doctors from SS group had experience of over 20 years. There was equal representation of clinicians from government and private settings in both SS and NSS group. There were $27.4 \%(n=442)$ corporate doctors in NSS group and $32.4 \%(n=558)$ in SS group. On an average about 150 patients were seen by both nonspecialist (mean 161.8) and specialist gastroenterologists (mean 151.1) in one week.

\section{APD patient profile}

Figure 2 shows the diagnosis and profile of the patients with APD treated by the clinicians. Amongst the patients diagnosed with APD, about $39 \%$ patients had GERD and $25 \%$ had non-ulcer dyspepsia (NUD). Of the $36 \%$ patients diagnosed with PUD, $10 \%$ had gastric ulcer and $20 \%$ had duodenal ulcer and approximately $16 \%$ patients were diagnosed with PUD without further classification as gastric or duodenal ulcer (PUD non-specified). The age distribution and the duration of clinical symptoms were almost similar in patients of GERD and PUD (Table 2). More than $70 \%$ of the responses suggested that patients having GERD and PUD were between 18 to 59 years of age. For both GERD and PUD, less than $25 \%$ of the cases were diagnosed within 1 month of onset of symptoms.

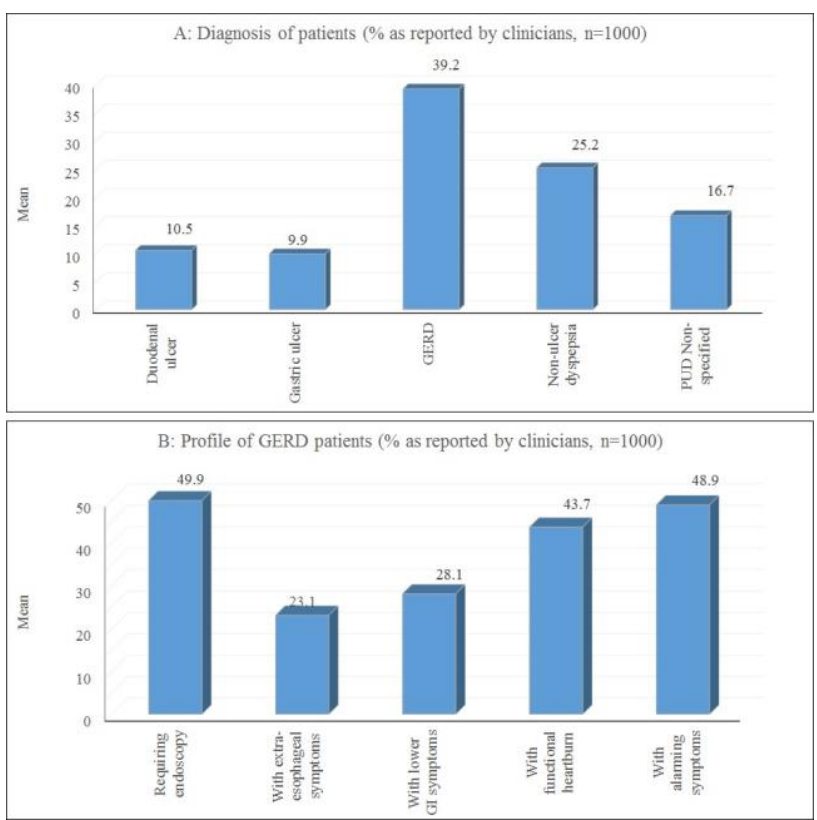

Figure 2: Diagnosis and profile of patients as reported by clinicians.

\section{Clinical presentation of APD}

The clinical presentation of APD is shown in Table 3. Heartburn and regurgitation were the main symptoms for which patients with GERD seeked medical attention. $60.5 \%$ of the responses ranked heartburn as the most common symptom in GERD. In at least $49 \%$ cases, the patient presented with an alarming symptom of GERD, the most common being dysphagia reported by $67 \%$ responses. Approximately $23 \%$ of patients with GERD had extra-esophageal symptoms, most common being reflux cough syndrome reported by $53 \%$ responses. $28 \%$ GERD patients had concomitant lower GI complaints like lower abdominal pain and constipation.

Table 2: Profile of patients with APD.

\begin{tabular}{|c|c|c|c|}
\hline \multirow{2}{*}{\multicolumn{2}{|c|}{ Diagnosis of patients (\%) }} & \multicolumn{2}{|l|}{ Mean } \\
\hline & & & \\
\hline \multicolumn{2}{|c|}{ - Duodenal ulcer } & \multicolumn{2}{|l|}{10.5} \\
\hline \multicolumn{2}{|c|}{ - Gastric ulcer } & \multicolumn{2}{|l|}{9.9} \\
\hline \multicolumn{2}{|l|}{ - GERD } & \multicolumn{2}{|l|}{39.2} \\
\hline \multicolumn{2}{|l|}{ - NUD } & \multicolumn{2}{|l|}{25.2} \\
\hline \multicolumn{2}{|c|}{ - PUD (Non-specified) } & \multicolumn{2}{|l|}{16.7} \\
\hline \multicolumn{2}{|c|}{$\begin{array}{l}\text { Gender distribution of } \\
\text { patients }\end{array}$} & \multicolumn{2}{|l|}{$\mathrm{M} / \mathrm{F}$} \\
\hline \multicolumn{2}{|c|}{ - Erosive GERD } & \multicolumn{2}{|l|}{$1 / 2$} \\
\hline \multicolumn{2}{|c|}{ - Non-erosive GERD } & \multicolumn{2}{|l|}{$1 / 2$} \\
\hline \multicolumn{2}{|c|}{$\begin{array}{l}\text { - PUD (Gastric ulcer and } \\
\text { Duodenal ulcer) }\end{array}$} & \multicolumn{2}{|l|}{$5 / 8$} \\
\hline \multicolumn{4}{|c|}{ - Profile of patients } \\
\hline \multicolumn{2}{|c|}{ - Requiring endoscopy } & \multicolumn{2}{|l|}{49.9} \\
\hline \multicolumn{2}{|c|}{$\begin{array}{l}\text { - With extra-esophageal } \\
\text { symptoms }\end{array}$} & \multicolumn{2}{|l|}{23.1} \\
\hline \multicolumn{2}{|c|}{ - With lower GI symptoms } & \multicolumn{2}{|l|}{28.1} \\
\hline \multicolumn{2}{|c|}{$\begin{array}{l}\text { With functional } \\
\text { heartburn }\end{array}$} & \multicolumn{2}{|l|}{43.7} \\
\hline - With alarm & g symptoms & 48.9 & \\
\hline & & GERD (\%) & PUD (\%) \\
\hline Age Distributic & of patients & & \\
\hline - $\quad<18$ years & Mean (SD) & $11.5(9.12)$ & $10.7(9.55)$ \\
\hline & Median & 10 & 10 \\
\hline $\begin{array}{l}18-39 \\
\text { years }\end{array}$ & Mean (SD) & $35.7(16.14)$ & $37.0(19.01)$ \\
\hline & Median & 35 & 35 \\
\hline $\begin{array}{ll}\text { - } & 40-59 \\
& \text { years }\end{array}$ & Mean (SD) & $34.2(14.53)$ & $33.1(14.39)$ \\
\hline & Median & 35 & 30 \\
\hline - $\geq 60$ years & Mean (SD) & $19.3(11.00)$ & $20.1(13.50)$ \\
\hline & Median & 20 & 20 \\
\hline Duration of cli & cal symptom & & \\
\hline - $\quad<1$ month & Mean (SD) & $21.9(17.70)$ & 23.3(18.09) \\
\hline & Median & 15 & 20 \\
\hline $\begin{array}{ll}-1-2 \\
\text { months }\end{array}$ & Mean (SD) & $25.8(14.19)$ & $28.1(15.38)$ \\
\hline & Median & 25 & 30 \\
\hline $\begin{array}{ll}- & 2-6 \\
& \text { months }\end{array}$ & Mean (SD) & $28.9(17.37)$ & $28.2(16.59)$ \\
\hline & Median & 30 & 25 \\
\hline - $>6$ months & Mean (SD) & $24.2(19.31)$ & $21.7(18.91)$ \\
\hline & Median & 20 & 15 \\
\hline
\end{tabular}


Table 3: Clinical presentation of patients with GERD and PUD.

\begin{tabular}{|c|c|c|c|c|c|c|}
\hline & \multicolumn{3}{|l|}{ GERD } & \multicolumn{3}{|l|}{ PUD } \\
\hline & $\begin{array}{l}\text { Non-specialists } \\
\text { (NS) } \\
\text { N }(\%)\end{array}$ & $\begin{array}{l}\text { Specialists } \\
\text { (SS) } \\
\mathbf{N}(\%)\end{array}$ & $\begin{array}{l}\text { Overall } \\
\mathbf{N}(\%)\end{array}$ & $\begin{array}{l}\text { Non- } \\
\text { specialists } \\
\text { (NS) } \\
\text { N }(\%)\end{array}$ & $\begin{array}{l}\text { Specialists } \\
\text { (SS) } \\
\mathbf{N}(\%)\end{array}$ & $\begin{array}{l}\text { Overall } \\
\mathbf{N}(\%)\end{array}$ \\
\hline $\mathbf{N}$ & 442 & 558 & 1000 & 442 & 558 & 1000 \\
\hline \multicolumn{7}{|c|}{ Most commonly encountered symptoms } \\
\hline Heartburn & $245(55.4)$ & $359(64.5)$ & $604(60.5)$ & - & - & - \\
\hline Regurgitation & $51(11.5)$ & $68(12.2)$ & $119(11.9)$ & - & - & - \\
\hline Epigastric pain & $62(14.0)$ & $55(9.9)$ & $117(11.7)$ & $316(71.5)$ & $406(72.9)$ & $722(72.3)$ \\
\hline Bloating and eructation & $72(16.3)$ & $79(14.2)$ & $151(15.1)$ & $31(7.0)$ & $40(7.2)$ & $71(7.1)$ \\
\hline Sleep disturbance & $26(5.9)$ & $19(3.4)$ & $45(4.5)$ & $17(3.8)$ & $18(3.2)$ & $35(3.5)$ \\
\hline Nausea and vomiting & - & - & - & $64(14.5)$ & $78(14.0)$ & $142(14.2)$ \\
\hline Pain awakening at night & - & - & - & $18(4.1)$ & $27(4.8)$ & $45(4.5)$ \\
\hline \multicolumn{7}{|c|}{ Most common alarming symptoms } \\
\hline Dysphagia & $294(66.5)$ & $377(67.8)$ & $671(67.4)$ & $46(10.4)$ & $26(4.8)$ & $72(7.4)$ \\
\hline Odynophagia & $46(10.4)$ & $49(8.8)$ & $95(9.5)$ & - & - & - \\
\hline GI bleeding & $30(6.8)$ & $48(8.6)$ & $78(7.8)$ & $209(47.3)$ & $265(47.7)$ & 474 (47.6) \\
\hline Weight loss & $24(5.4)$ & $31(5.6)$ & $55(5.5)$ & $33(7.5)$ & $36(6.5)$ & $69(6.9)$ \\
\hline Anemia & $12(2.7)$ & $15(2.7)$ & $27(2.7)$ & $51(11.5)$ & $105(18.9)$ & $156(15.7)$ \\
\hline Persistent vomiting & - & - & - & $80(18.1)$ & $88(15.8)$ & $168(16.9)$ \\
\hline Jaundice & - & - & - & $7(1.6)$ & $9(1.7)$ & $16(1.7)$ \\
\hline \multicolumn{7}{|c|}{ Commonly encountered symptoms related to delayed gastric emptying in GERD } \\
\hline Nausea & $126(28.5)$ & $136(24.4)$ & $262(26.2)$ & & & \\
\hline Vomiting & $30(6.8)$ & $47(8.4)$ & 77 ( 7.7) & & & \\
\hline Early satiety & $75(17.0)$ & $123(22.1)$ & $198(19.8)$ & & & \\
\hline Abdominal discomfort & $170(38.5)$ & $167(30.0)$ & $337(33.7)$ & & & \\
\hline Post-prandial fullness & 48 (10.9) & $104(18.7)$ & $152(15.2)$ & & & \\
\hline \multicolumn{7}{|c|}{ Commonly encountered extra-oesophageal symptoms in GERD } \\
\hline Reflux cough syndrome & $235(53.2)$ & $296(53.2)$ & $531(53.3)$ & & & \\
\hline Reflux asthma syndrome & $31(7.0)$ & $54(9.7)$ & 85 ( 8.6) & & & \\
\hline Reflux laryngitis syndrome & 78 (17.6) & $74(13.3)$ & $152(15.2)$ & & & \\
\hline Pharyngitis & $78(17.6)$ & $122(22.0)$ & $200(20.2)$ & & & \\
\hline Recurrent sinusitis & $8(1.8)$ & $7(1.3)$ & $15(1.5)$ & & & \\
\hline Recurrent otitis media & $0(0.0)$ & $7(1.3)$ & $7(0.7)$ & & & \\
\hline Idiopathic pulmonary fibrosis & $6(1.4)$ & $9(1.6)$ & $15(1.5)$ & & & \\
\hline
\end{tabular}

Abdominal discomfort and postprandial fullness was the most common symptom related to delay gastric emptying (33.7\% responses). $72.3 \%$ of the responses ranked 'epigastric pain' as the most common symptom in PUD. Nausea and vomiting were also reported in PUD by $14.2 \%$ responses and these symptoms were not reported by GERD patients. Other symptoms reported by PUD patients were bloating and eructation $(7.1 \%)$, sleep disturbances (3.5\%) and night awakenings (4.5\%). Gastro-intestinal bleeding was the common alarming symptom reported by $47.6 \%$ responses (equally reported by both SS and NSS clinicians). Other alarming symptoms in PUD included persistent vomiting, anemia, dysphagia and weight loss.

\section{Diagnostic modalities in APD}

The diagnosis of GERD was primarily based on the clinical symptoms in majority of patients as $78.9 \%$ of the responses ranked 'clinical symptoms' as the most common diagnostic tool; $10.2 \%$ responses were using an empirical therapy with a proton pump inhibitor (PPI) to establish clinical diagnosis (Table 4). It is noted that more non-specialists compared with specialists used GERD specific questionnaire to confirm diagnosis of GERD. Similarly, the diagnosis of PUD was primarily based on clinical symptoms as per $64.0 \%$ responses. Also, more specialists advocate esophagogastroduodenoscopy (32.4\%) than non-specialists $(19.5 \%)$ as a diagnostic tool for APD. Almost $50 \%$ of the cases diagnosed as APD required an upper GI endoscopy. New onset dyspepsia in 
patients above 50 years of age was a most common indication for upper GI endoscopy mentioned by $40.3 \%$ responses. Almost $43 \%$ patients showed negative finding on the upper GI endoscopy and were also negative for 24hour esophageal $\mathrm{pH}$ testing, diagnosed as 'functional heartburn'. Failure of PPI therapy was the most common indication for esophageal $\mathrm{pH}$ monitoring highlighted by $64.2 \%$ responses. Esophageal manometry was primarily used as a diagnostic tool of choice for esophageal motility disorders by $55.2 \%$ responses.

Table 4: Diagnostic modalities in patients of APD.

\begin{tabular}{|c|c|c|c|}
\hline & $\begin{array}{l}\text { Non-specialists } \\
\text { (NS) } \\
\mathbf{N}(\%)\end{array}$ & $\begin{array}{l}\text { Specialists } \\
\text { (SS) } \\
\mathbf{N}(\%)\end{array}$ & $\begin{array}{l}\text { Overall } \\
\mathbf{N}(\%)\end{array}$ \\
\hline & 442 & 558 & 1000 \\
\hline \multicolumn{4}{|l|}{ Diagnostic tool used } \\
\hline - Clinical symptoms & $361(81.7)$ & $425(76.2)$ & $786(78.6)$ \\
\hline - Empirical trial of PPI & $44(10.0)$ & $58(10.4)$ & $102(10.2)$ \\
\hline - Questionnaire for GERD & $37(8.4)$ & $26(4.7)$ & $63(6.3)$ \\
\hline - Esophagogastroduodenoscopy & $86(19.5)$ & $181(32.4)$ & $69(6.9)$ \\
\hline - 24 hour $\mathrm{pH}$ monitoring & $6(1.4)$ & $12(2.2)$ & $18(1.8)$ \\
\hline - H.pylori testing & $18(4.1)$ & $14(2.5)$ & $32(3.2)$ \\
\hline - Radiological imaging & $4(0.9)$ & $5(0.9)$ & $9(0.9)$ \\
\hline \multicolumn{4}{|l|}{ Indication for Upper GI Endoscopy } \\
\hline - Age $>50$ years with new onset dyspepsia & $149(33.7)$ & $252(45.3)$ & $401(40.3)$ \\
\hline - Alarming symptoms of GERD and PUD & $178(40.3)$ & $175(31.5)$ & $353(35.4)$ \\
\hline - High risk for structural disease or malignancy & $63(14.3)$ & $69(12.4)$ & $132(13.2)$ \\
\hline - Persistence of dyspeptic symptoms & $78(17.6)$ & $70(12.6)$ & $148(14.8)$ \\
\hline - For confirmation of diagnosis & $46(10.4)$ & $40(7.2)$ & $86(8.6)$ \\
\hline \multicolumn{4}{|l|}{ Indication for Esophageal pH monitoring study } \\
\hline - Failure of PPI therapy & $269(60.9)$ & $369(66.7)$ & $638(64.4)$ \\
\hline - Preoperative assessment & $79(17.9)$ & $105(19.0)$ & $184(18.6)$ \\
\hline - Patient with persistent reflux symptoms despite anti-reflux surgery & $87(19.7)$ & $85(15.4)$ & $172(17.4)$ \\
\hline \multicolumn{4}{|l|}{ - Indication for esophageal manometry } \\
\hline $\begin{array}{l}\text { - To reassess esophageal function in patients treated for esophageal } \\
\text { disorder }\end{array}$ & $116(26.2)$ & $131(23.9)$ & $247(25.4)$ \\
\hline - To diagnose primary esophageal motility disorders & $217(49.1)$ & $318(58.1)$ & $535(55.2)$ \\
\hline $\begin{array}{l}\text { - To diagnose secondary esophageal motility disorders with systemic } \\
\text { disease }\end{array}$ & $31(7.0)$ & $37(6.8)$ & $68(7.0)$ \\
\hline - Pre-operative assessment of patients undergoing anti-reflux procedure & $45(10.2)$ & $53(9.7)$ & $98(10.1)$ \\
\hline - To guide placement of $\mathrm{pH}$ electrode for ambulatory $\mathrm{pH}$ monitoring & $12(2.7)$ & $19(3.5)$ & $31(3.2)$ \\
\hline
\end{tabular}

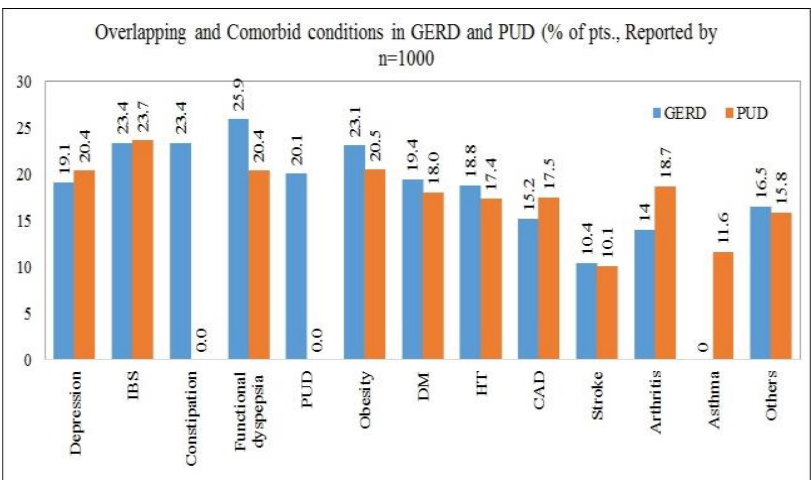

CAD: coronary artery disease; DM: diabetes mellitus; GERD: gastroesophageal reflux disease; HT: Hypertension; IBS: irritable bowel syndrome; PUD: peptic ulcer disease

Figure 3: Overlapping and comorbid conditions in GERD and PUD.

\section{Overlapping conditions with GERD and PUD}

Figure 3 presents the overlapping conditions in patients with GERD and PUD. GERD was commonly associated with Functional dyspepsia (25.9\%), irritable bowel syndrome $(23.4 \%)$ and constipation $(23.4 \%)$ while IBS; $23.7 \%$ ), obesity $(20.5 \%)$, depression $(20 \%)$, functional dyspepsia (20.4\%) were the commonly occurring overlapping conditions associated with PUD.

\section{DISCUSSION}

Acid peptic disorders are caused by various pathogenic mechanisms involving either excessive acid secretion and/or diminished mucosal defense. ${ }^{10}$ Since they are common conditions present in daily clinical practice, due to their chronicity, they represent a significant cost to 
healthcare. Acid reflux in GERD causes damage to the esophageal mucosa, potentially causing laryngeal tissue injury with subsequent development of pulmonary symptoms. ${ }^{11}$

The present survey study aimed to understand the epidemiology, clinical presentation and associated overlapping conditions in patients with APD in Indian setting. About $39.2 \%$ patients were reported to have GERD and $37.1 \%$ patients had PUD (duodenal ulcer $10.5 \%$, gastric ulcer $9.9 \%$ and peptic ulcer non-specified $16.7 \%$ ), whereas $25.2 \%$ patients were reported to have non-ulcer dyspepsia. These observations were consistent with another study involving 2700 patients with dyspeptic symptoms referred for open access upper endoscopy in which $45 \%$ of subjects had PUD. ${ }^{12}$ Presently, reflux esophagitis is the most common acid peptic disorder reported compared to peptic ulcer which was the most common APD previously. ${ }^{13}$ This may also be true for Indian population due to significant alterations in lifestyle habits, such as more westernized dietary habits, and environmental factors including improved hygiene. ${ }^{14}$

The most common age group observed for GERD was 18-39 years (35.7\%) followed by $40-59$ years $(34.2 \%)$, which is in line with a report by Thrift et al who reported onset of GERD in patients below 30 years. ${ }^{15}$ Similarly, the most common age group for PUD was 18-39 years (37.0\%) followed by $40-59$ years $(33.1 \%)$. A 10-year follow-up study based on a structured telephone interview in 728 patients with an endoscopic diagnosis of peptic ulcer reported that even after prolonged therapy, more than one-third of the patients with PUD are still burdened with symptoms and complications of PUD. $1^{6}$ Also, PUD is often associated with Helicobacter pylori infection and use of non-steroidal anti-inflammatory agents.6 Hence PUD may have great impact on the quality of life, especially in younger age group. Current observations show that the patients with both GERD and PUD had a variable duration of clinical symptoms ranging from $<1$ month to $>6$ months. The risk of development of Barrett's esophagus has shown to increase with earlier onset age of frequent GERD symptoms and chronicity of symptoms. ${ }^{15}$ Thus, the age at presentation and chronicity of symptoms may facilitate clinicians in endoscopy decisions, to rule out BE.

Clinical symptoms are the most common diagnostic criteria for APD used by clinicians, both specialists $(76.2 \%)$ and non-specialists $(81.7 \%)$. Other than the clinical symptoms, other diagnostic criteria used are the questionnaire for GERD (6.3\%), esophagogastroduodenoscopy (6.9\%), 24-hour $\mathrm{pH}$ monitoring $(<2 \%), \quad H$. pylori testing $(3.2 \%)$ and radiological imaging $(<1 \%)$. However, endoscopy remains one of the important tools for diagnosis of APD. ${ }^{17}$ This study highlights that the most common indication for $\mathrm{pH}$ monitoring is failure of PPI therapy (64.4\% clinicians) and a preoperative assessment (18.6\% clinicians). $\mathrm{pH}$ monitoring is also recommended in patients with persistent reflux symptoms despite antireflux surgery by $17.45 \%$ clinicians. Esophageal manometry is most commonly used for diagnosis of primary esophageal motility disorders ( $55.2 \%$ clinicians) and to reassess the esophageal function in patients treated for esophageal disorder ( $25.4 \%$ clinicians). Esophageal manometry is sometimes used for pre-operative assessment of patients undergoing anti-reflux procedure $(10.1 \%)$ and to guide the placement of $\mathrm{pH}$ electrode for ambulatory $\mathrm{pH}$ monitoring (3.2\% clinicians). Patients with GERD and PUD often present with extra-intestinal symptoms like chronic laryngitis, asthma and pseudoangina. ${ }^{18}$ Also, many patients with GERD present with otorhinolaryngology symptoms and these patients land up being treated with medications based on the findings of nasal endoscopy and laryngoscopy, since they consult only an ENT surgeon. ${ }^{19}$ Hence, it has been recommended that these patients are to be screened using esophageal manometry and $\mathrm{pH}$ monitoring to ensure correct line of management. ${ }^{20}$

About $60.5 \%$ of the responses ranked heartburn as a most common symptom (Rank 1) in GERD. This finding is like the one indicated by Quigley EM as the most common symptom in GERD. ${ }^{21}$ Similarly, $72.3 \%$ of the responses ranked 'epigastric pain' as a most common symptom (Rank 1) in PUD, which is similar the reported data by Najm WL (2011) in PUD patients. ${ }^{22}$ Also, a systematic review done to understand the burden of PUD reports $81 \%$ patients with epigastric pain and diffuse abdominal pain. ${ }^{23}$ In the present study, $33.7 \%$ of the responses ranked 'postprandial abdominal discomfort' as the most commonly encountered symptom related to gastric emptying in GERD. In our study, clinical symptoms were the most common diagnostic tool for GERD (78.9\% responses), which agrees with the data reported by Katz PO et al. ${ }^{24}$ However, it should be considered that with increasing age, the incidence of musculoskeletal and cardiovascular disorders also increase leading to subsequent increase in non-steroidal anti-inflammatory use and consequent increase in APD. Thus, causes and presenting patterns of APD may change with advance age. ${ }^{25,26}$ Dysphagia was the most alarming symptom of GERD reported for $67.4 \%$ of the responses, whereas for PUD, GI bleeding was the most common alarming symptom (47.6\% responses). The profile of patients visiting the specialist gastroenterologists and non-specialist doctors seem to be similar with respect to the presenting symptoms and associated conditions.

Most common overlapping conditions with GERD were functional dyspepsia $(25.9 \%)$, constipation $(23.4 \%)$ and irritable bowel syndrome $(23.4 \%)$. On the other hand, in PUD, the most common overlapping conditions reported were functional dyspepsia $(20.4 \%)$, obesity $(20.5 \%)$ and irritable bowel syndrome $(20.4 \%)$. Hence, these are the symptoms to be looked for by clinicians during diagnosis and management of GERD and PUD. It is important to look for specific symptoms since GERD is reported to be 
usually over diagnosed and over treated. ${ }^{27}$ Although, IBS and GERD affect different regions of the intestines, significant overlap in these two conditions has been well documented. ${ }^{28}$ Other comorbid conditions like arthritis, diabetes mellitus, coronary artery disease, depression, hypertension and asthma were also commonly observed in patients of APD. Published literature suggests that as many as $75 \%$ of patients visiting diabetic clinics report significant GI symptoms. ${ }^{29}$ Type II diabetes mellitus has been described as a possible risk factor for the development of GERD. In this survey, approximately $20 \%$ of the cases were associated with diabetes mellitus, in line with earlier studies in Asian population indicating a prevalence of approximately $18-25 \% .^{29}$ Arthritic patients usually receive anti-inflammatory agents like COX inhibitors or corticosteroids and hence are more prone for PUD and GERD. Similarly, patients with cardiovascular disorders are usually on aspirin therapy, predisposing them to APD. Although sleep disturbance was reported to be below $5 \%$ in this study by both specialist and non-specialist clinicians, there are various studies which point towards a greater prevalence of sleep disturbance symptoms in GERD ${ }^{10}$ and PUD, with many patients having sleep apnea syndrome. ${ }^{30,31}$ This could be possibly due to the nature of the study which involved data collection from clinicians based on their memory recall and judgment. Recently published article on analysis of data of 10029 endoscopies conducted revealed changing trends in the incidence of various pathologies of the upper gastrointestinal tract, thus calling for periodic review of epidemiology of APD in India. ${ }^{8}$

This study has several merits. Firstly, this study highlights the clinical profiling of APD patients across the country. Secondly, this study gives insights into clinical presentation and diagnosis of patients of APD by specialist and non-specialist clinicians. Further, this also gives insights into diagnosis of the overlapping and comorbid conditions associated with APD in clinical practice. This study being a survey of the clinician's response based on their recall of information, there is a scope for errors in terms of data accuracy and possibilities of incorrect information.

\section{CONCLUSION}

APD is more common in age group 18-59 years with heart burn as the most common symptom for GERD and epigastric pain as the most common symptom for PUD. Diagnosis is mostly based on clinical symptoms and nearly $50 \%$ patients of APD cases present with alarming symptoms and require prompt endoscopy. Hence, there is need to focus on these overlapping conditions while managing patients of APD.

\section{ACKNOWLEDGEMENTS}

The authors would like to acknowledge Dr. Deepak Langade for his contribution in writing the manuscript.
Funding: Funded by Abbott India Ltd.

Conflict of interest: Dr. Rooprai has received research funding from Abbott India Ltd. Dr. Gangadhar and Dr. Mayabhate are employees of Abbott. All authors have indicated that they have no other conflict of interest regarding the outcome of this study

Ethical approval: The study was approved by the Institutional Ethics Committee

\section{REFERENCES}

1. Shin JM, Vagin O, Munson K, Kidd M, Modlin IM, Sachs G. Molecular mechanisms in therapy of acidrelated diseases. Cell Mol Life Sci. 2008;65(2):26481.

2. Dutta AK, Chacko A, Balekuduru A, Sahu MK, Gangadharan SK. Time trends in epidemiology of peptic ulcer disease in India over two decades. Indian J Gastroenterol. 2012;31(3):111-5.

3. Bhatia SJ, Reddy DN, Ghoshal UC. Epidemiology and symptom profile of gastroesophageal reflux in the Indian population: report of the Indian Society of Gastroenterology Task Force. Indian J Gastroenterol. 2011;30(3):118-27.

4. Rameschandra S, Acharya V, Kunal, Vishwanath T, Ramkrishna A, Acharya P. Prevalence and Spectrum of Gastro Esophageal Reflux Disease in Bronchial Asthma. J Clin Diagn Res. 2015;9(10):OC11-4.

5. Boeckxstaens GE, Rohof WO. Pathophysiology of gastroesophageal reflux disease. Gastroenterol Clin North Am. 2014;43(1):15-25.

6. Sung JJY, Kuipers EJ, El-Serag HB. Systematic review: the global incidence and prevalence of peptic ulcer disease. Aliment Pharmacol Ther. 2009;29(9):938-46.

7. Aro P, Storskrubb T, Ronkainen J. Peptic ulcer disease in a general adult population: the Kalixanda study: a random population-based study. Am J Epidemiol. 2006;163(11):1025-34.

8. Mohiuddin MK, Chowdavaram S, Bogadi V. Epidemic Trends of Upper Gastrointestinal Tract Abnormalities: Hospital-based study on Endoscopic Data Evaluation. Asian Pac J Cancer Prev. 2015;16(14):5741-7.

http://www.ncbi.nlm.nih.gov/pubmed/26320445. Accessed March 1, 2016.

9. Singh VK, Kathiresan P, Kathiresa NP, Singh PK. Prescribing pattern of acid suppressants in modern clinical practice- An analysis. Pharm Sin. 2011;2(3):67-73.

10. Moayyedi P, Hunt R, Armstrong D, Lei Y, Bukoski M, White R. The impact of intensifying acid suppression on sleep disturbance related to gastrooesophageal reflux disease in primary care. Aliment Pharmacol Ther. 2013;37(7):730-7.

11. Mejia A, Kraft WK. Acid peptic diseases: pharmacological approach to treatment. Expert Rev Clin Pharmacol. 2009;2(3):295-314.

12. Xia B, Xia HHX, Ma CW. Trends in the prevalence of peptic ulcer disease and Helicobacter pylori 
infection in family physician-referred uninvestigated dyspeptic patients in Hong Kong. Aliment Pharmacol Ther. 2005;22(3):243-9.

13. Haruma $K$, Kamada $T$, Oosawa M, Murao $T$, Shiotani A. [Acid related diseases--comparison between western countries and Japan]. Nihon Rinsho. 2015;73(7):1081-5.

http://www.ncbi.nlm.nih.gov/pubmed/26165061.

Accessed June 24, 2016.

14. Furuta K, Kinoshita Y. [Relationship of acid-related diseases with lifestyle habit changes and environmental factors]. Nihon Rinsho. 2015;73(7):1086-92.

http://www.ncbi.nlm.nih.gov/pubmed/26165062. Accessed June 24, 2016.

15. Thrift AP, Kramer JR, Qureshi Z, Richardson PA, ElSerag HB. Age at onset of GERD symptoms predicts risk of Barrett's esophagus. Am J Gastroenterol. 2013;108(6):915-22.

16. Petersen H, Kristensen P, Johannessen T, Kleveland PM, Dybdahl JH, Myrvold H. The natural course of peptic ulcer disease and its predictors. Scand J Gastroenterol. 1995;30(1):17-24. http://www.ncbi.nlm.nih.gov/pubmed/7701245. Accessed March 6, 2016.

17. Triadafilopoulos G. Endoscopic Options for Gastroesophageal Reflux: Where Are We Now and What Does the Future Hold? Curr Gastroenterol Rep. 2016;18(9):47.

18. Bărboi OB, Prelipcean CC, Mihai C. Extra digestive manifestations of gastroesophageal reflux disease: demographic, clinical, biological and endoscopic features. Rev medico-chirurgicală a Soc Medici şi Nat din Iaşi. 2016;120(2):282-7.

19. Roman S, Holloway R, Keller J. Validation of criteria for the definition of transient lower esophageal sphincter relaxations using high-resolution manometry. Neurogastroenterol Motil. 2016.

20. Herbella F, Andolfi C, Vigneswaran Y, Patti M, Pinna B. Importance of esophageal manometry and $\mathrm{pH}$ monitoring for the evaluation of otorhinolaryngologic (ENT) manifestations of GERD. A multicenter study. J Gastrointest Surg. 2016.
21. Quigley EMM. Non-erosive reflux disease, functional heartburn and gastroesophageal reflux disease; insights into pathophysiology and clinical presentation. Chin J Dig Dis. 2006;7(4):186-90.

22. Najm WI. Peptic ulcer disease. Prim Care. 2011;38(3):383-94.

23. Barkun A, Leontiadis G. Systematic review of the symptom burden, quality of life impairment and costs associated with peptic ulcer disease. Am J Med. 2010;123(4):358-66.

24. Katz PO, Gerson LB, Vela MF. Guidelines for the Diagnosis and Management of Gastroesophageal Reflux Disease. Am J Gastroenterol. 2013;108(3):308-28.

25. An JH, Kim SG. L-carnitine supplementation in hypothyroidism [Letter to the Editor]. Endocr J. 2016;63(10):939-40.

26. Kim BW. [Diagnosis and Treatment of Peptic Ulcer Disease: Present and Future Perspective]. Korean J Gastroenterol. 2016;67(6):318-20.

27. Wilson JL, Pruett KL. Gastroesophageal Reflux Disease: Treating Wisely. N C Med J. 2016;77(3):202-205.

28. Bortoli $\mathrm{N}$ de. Overlap of functional heartburn and gastroesophageal reflux disease with irritable bowel syndrome. World J Gastroenterol. 2013;19(35):5787.

29. Sun H, Yi L, Wu P. Prevalence of Gastroesophageal Reflux Disease in Type II Diabetes Mellitus. Gastroenterol Res Pract. 2014;(601571):1-4.

30. Shiao TH, Liu CJ, Luo JC. Sleep apnea and risk of peptic ulcer bleeding: a nationwide population-based study. Am J Med. 2013;126(3):249-55.

31. Qu Y, Ye JY, Han DM. Esophageal Functional Changes in Obstructive Sleep Apnea/Hypopnea Syndrome and Their Impact on Laryngopharyngeal Reflux Disease. Chin Med J (Engl). 2015;128(16):2162-7.

Cite this article as: Rai RR, Gangadhar A, Mayabhate MM. Clinical profiling of patients with Acid Peptic Disorders (APD) in India: a crosssectional survey of clinicians. Int J Basic Clin Pharmacol 2017;6:194-202. 